\title{
Linear Dichroism in the NEXAFS Spectra of $n$-Alkane Crystalline Polymorphs
}

Sahan D. Perera ${ }^{1}$, Jian Wang ${ }^{2}$, Stephen G. Urquhart ${ }^{1 *}$

1. Department of Chemistry, University of Saskatchewan, Saskatoon, SK, Treaty Six Territory, Canada S7N 5C9

2. Canadian Light Source, University of Saskatchewan, Saskatoon, SK, Canada S7N 0X4

\begin{abstract}
Linear Dichroism in Near Edge X-ray Absorption Fine Structure (NEXAFS) spectroscopy is a useful tool for studying molecular orientation and for clarifying spectroscopic assignments. $n$ Alkane molecules can form single crystals with different polymorphs; orthorhombic and monoclinic in the case of even chain lengths between $n-\mathrm{C}_{28} \mathrm{H}_{58}$ and $n-\mathrm{C}_{40} \mathrm{H}_{82}$. These polymorphs exhibit different linear dichroism in their NEXAFS spectra; in particular a backbone-oriented transition contributes to the low-energy " $\mathrm{C}-\mathrm{H}$ " band in the monoclinic polymorph.
\end{abstract}

* Author to whom correspondence should be addressed. E-mail: stephen.urquhart@usask.ca 


\section{Introduction}

$n$-Alkanes $\left(\mathrm{C}_{\mathrm{n}} \mathrm{H}_{2 \mathrm{n}+2}\right)$ are simple building blocks of complex molecules that include oils, liquid crystals, and polymers. Although $n$-alkanes have a simple structure built upon methyl and methylene groups, the Near Edge X-ray Absorption Fine Structure (NEXAFS) spectra of $n$-alkanes have significant complexity. These spectra vary with the crystalline polymorph, chain length and degree of disorder.

NEXAFS spectroscopy is often used to study the chemical composition[1-7] and bonding[1, 8-10] in organic molecules. In NEXAFS spectroscopy, spectral features are often described as one electron transitions from core electron (e.g. carbon 1s) to an unoccupied valence molecular orbital (e.g. a carbon $1 \mathrm{~s} \rightarrow \sigma^{*}$ or a carbon $1 \mathrm{~s} \rightarrow \pi^{*}$ transition), to Rydberg orbitals (e.g. a carbon $1 \mathrm{~s} \rightarrow 3 \mathrm{p}$ transition), or to transitions with mixed Rydberg / valence character.[11-13] The intensities of these resonances depend on the angle $(\theta)$ between the electric field vector of linearly polarized X-rays $(E)$ and the transition dipole moment (TDM) for a particular transition. The angle dependence of these resonances follows Equation 1:

$$
\mathrm{I} \propto\left|E \cdot \mu_{\mathrm{if}}\right|^{2} \propto \cos ^{2} \theta
$$

Where $\mu_{\mathrm{if}}$ is the TDM for the one electron transition from the initial state $\psi_{\mathrm{i}}$ to the final state $\psi_{\mathrm{f}}$ :

$\mu_{\mathrm{if}}=\left\langle\psi_{\mathrm{f}}|\mu| \psi_{\mathrm{i}}\right\rangle$,

where $\mu$ is electric dipole operator. The maximum absorption intensity occurs when the TDM of a NEXAFS transition is parallel to the electric field vector $(E)$. This linear dichroism (LD) can be used to study molecular orientation for $n$-alkanes and other molecular species and can help clarify spectroscopic assignments.[14-23]

The morphology of an $n$-alkane sample and the orientation of $n$-alkane chains within this sample are affected by conditions that include $n$-alkane chain length,[24] substrate temperature during growth, and the sample preparation method (e.g. solution casting versus epitaxial growth by physical vapour deposition).[21, 23-26] Semi-ordered films can be grown by casting dilute $n$ alkane solutions onto a surface; these generally have their $n$-alkane chains oriented normal to the surface or at some tilt angle, with rotational disorder about the surface normal. In-plane oriented $n$-alkane thin films can be prepared by epitaxial growth on high symmetry substrates. For example, $n$-alkane films deposited on $\mathrm{NaCl}(001)$ or $\mathrm{HOPG}(0001)$ surfaces grow with an in-plane orientation 
that depends on the surface symmetry, with crystalline morphologies that depend on chain length and substrate temperature.[19, 24, 27] Endo et al.[28] have used angle dependent NEXAFS spectroscopy to characterize "edge-on" and "side-on" geometries of in-plane oriented $n-\mathrm{C}_{12} \mathrm{H}_{26}$ on HOPG(0001) as a function of deposition thicknesses. As the film thickness increased, the $n$-alkane chain orientation changed from a geometry with the C-C-C plane edge-on to the surface to a geometry with the C-C-C plane 'side-on', that is, with the C-C-C plane parallel to the substrate plane.[28]

$n$-Alkane molecules can be crystalized in a regular diamond or parallelogram shape with straight edges, where the $n$-alkane chains within the crystal are directed $\sim$ perpendicular to the lamella surface.[23, 29] At room temperature, higher even $n$-alkanes $(\mathrm{n}>26)$ exhibit two different crystalline polymorphs: orthorhombic (o-rh) and monoclinic (mon).[29] For a given alkane chain length, both polymorphs can be formed. Plomp et al.[30] found that the metastable orthorhombic polymorph is formed instead of the stable monoclinic form when impurities consisting of $n$-alkanes with shorter chain lengths were present.[30] Solvent selection can also affect the formation of polymorphic structures for given alkane chain length.[31]

The structural difference between monoclinic and orthorhombic polymorphs of a given $n$-alkane is the way that $n$-alkane molecules stack within a layer.[30, 32, 33] In the orthorhombic polymorph (Pca21),[34] $n$-alkane chains are oriented normal to the substrate $(\mathrm{a}, \mathrm{b})$ plane, while in the monoclinic polymorph $\left(\mathrm{P} 2{ }_{1} / \mathrm{a}\right),[35] n$-alkane chains are tilted with an angle of about $61-65^{\circ}$ with respect to the substrate (a,b) plane (Scheme 1).[30, 32, 35, 36] A third possible orthorhombic polymorph (polytypic; Pcab,[30] not shown in Scheme 1) consists of two alternating tilted layers - each layer individually like the monoclinic polymorph, but together forming an orthorhombic structure.[30, 37, 38] The orthorhombic and monoclinic polymorphs can be resolved by optical microscopy, and therefore provide excellent structural models for single-crystal resolved NEXAFS spectroscopy studies.

Broadly, the NEXAFS spectra of $n$-alkanes consists of a low energy " $\mathrm{C}-\mathrm{H}$ " band (287-288 eV) and higher energy carbon $1 \mathrm{~s} \rightarrow \sigma^{*}$ C-c transitions (295-305 eV).[21] The LD of the higher energy carbon 1s $\rightarrow \sigma^{*}{ }_{\mathrm{C}-\mathrm{C}}$ band is the subject of a long-standing controversy between a "building block" model that treats the molecule's electronic structure as an assembly of bond-specific $\sigma^{*}{ }_{\mathrm{C}-\mathrm{C}}$ orbitals[37] and a molecular-orbital (MO) model[38] that recognizes that MOs are not localized to atom pairs, 
but are delocalized along the $n$-alkane backbone. When LD is used for orientation measurements, these contrasting models provide different descriptions of the molecular orientation; the $\cos ^{2} \theta$ dependence means that there is no angle where the carbon $1 \mathrm{~s} \rightarrow \sigma^{*}{ }_{\mathrm{C}-\mathrm{C}}$ transition intensity would go to zero in the "building block" model, while the intensity of this transition goes to zero in the molecular orbital model.[21] Molecular disorder could be misinterpreted as evidence for the "building block" model, as it would have the same effect on the experimental data. Fu et al[21] examined the angle resolved NEXAFS spectra of laterally oriented $n$-alkane single crystals, and found that their LD results corresponded to the molecular orbital model, explicitly excluding the "building block" model.[21]

This work is focused on the low energy "C-H" band (287-288 eV) in the NEXAFS spectra of $n$ alkanes. This band shows strong LD effects. The TDM for the primary "C-H" band spectroscopic features is directed in the $\mathrm{CH}_{2}$ plane of the methylene group, normal to the chain axis.[21] The polarization dependence of these " $\mathrm{C}-\mathrm{H}$ " band transitions has been explored by several groups; [28, 39] we will use the notion of Endo et al.,[28] as illustrated in Scheme 2. The first resonance is assigned as the carbon $1 \mathrm{~s} \rightarrow \sigma^{*} \mathrm{C}-\mathrm{H} / R_{\|}$transition, with its TDM within the C-C-C plane but perpendicular to the $n$-alkane long axis (e.g. parallel to the C-C-C plane).[28] The second resonance is assigned as the carbon $1 \mathrm{~s} \rightarrow \sigma^{*} \mathrm{C}-\mathrm{H} / R_{\perp}$ transition, with its TDM is perpendicular to both the $\mathrm{C}$-C-C plane and to the $n$-alkane long axis (e.g. $\perp$ to the $\mathrm{C}$-C-C plane).[28] These assignments are based on the geometry of the individual $n$-alkane molecule. In contrast to this molecular view, Zou et al. [25] proposed that the LD in these " $\mathrm{C}-\mathrm{H}$ " band transitions reflects the symmetry of the crystalline sample rather than of a collection of individual molecules. The origin of their proposed breakdown of the individual molecule model has not yet been clarified by theory. Swaraj et al.[26] examined the variation in the carbon 1s NEXAFS spectra as a function of $n$ alkane chain length and the parity of the carbon back bone (e.g. odd and even chain lengths). They proposed that the pre-edge "C-H" band features are affected by the parity (e.g. even/odd chain length) of the carbon backbone, but not the $n$-alkane chain length. This work did not account the crystal polymorphism in the $n$-alkane single crystals. As NEXAFS is sensitive to orientation, order, density[40] and crystal symmetry,[25] the crystal polymorph should also be important in determining the NEXAFS spectra and angle dependence for $n$-alkane single crystals 
In their study on laterally oriented $n$-alkane single crystals, Fu and Urquhart observed a backboneoriented transition $\sim 30 \mathrm{meV}$ above the second peak in the " $\mathrm{C}-\mathrm{H}$ " band, above the carbon $1 \mathrm{~s} \rightarrow$ $\sigma^{*}{ }_{\mathrm{C}-\mathrm{H}} / R_{\perp}$ transition, at $288.51 \mathrm{eV}$ in $n$-hexatriacontane, $n-\mathrm{C}_{60} \mathrm{H}_{122}$.[21] This low-energy backbone oriented transition was observed in addition to the traditionally assigned carbon $1 \mathrm{~s} \rightarrow \sigma^{*}{ }_{\mathrm{C}-\mathrm{C}}$ backbone oriented transition at higher energy (295-305 eV).[21] This observation goes against the common conception of the LD NEXAFS spectra of $n$-alkanes, where the low energy " $\mathrm{C}$-H" band was seen only as having a polarization normal to the chain axis, in the $\mathrm{CH}_{2}$ plane.

Scanning Transmission X-ray Microscopy (STXM)[9, 41] is useful for these NEXAFS spectroscopy studies, as individual $n$-alkane single crystals can be individually identified and examined. The typical experimental geometry in a STXM is normal incidence to planar substrate. For orthorhombic crystals, the X-ray electric field vector $(E)$ will be projected in the crystal $(\mathrm{a}, \mathrm{b})$ plane, whereas for monoclinic crystals, the electric field vector $(E)$ will also have a weak projection along the c axis of the crystal, or along the $n$-alkane molecular backbone.

To better understand the NEXAFS spectra of $n$-alkane single crystals and to clarify assignments, we have examined the LD in the carbon 1s NEXAFS spectroscopy of several linear $n$-alkanes $(n$ $\mathrm{C}_{28} \mathrm{H}_{58}, n-\mathrm{C}_{32} \mathrm{H}_{66}$ and $n-\mathrm{C}_{40} \mathrm{H}_{82}$ ), for different well-resolved crystal structures (orthorhombic and monoclinic). Systematic differences with chain length and crystal structure are characterized and interpreted.

\section{Experimental Section}

\subsection{Samples and Sample Preparation.}

Samples: Linear alkanes used in this study are $n$-tetracontane $\left(n-\mathrm{C}_{40} \mathrm{H}_{82}, 97+\%\right), n$-dotriacontane $\left(n-\mathrm{C}_{32} \mathrm{H}_{66}, 98 \%\right)$ and $n$-octacosane $\left(n-\mathrm{C}_{28} \mathrm{H}_{58}, 99 \%\right)$. These were purchased from Alfa Aesar and used without further purification. All the solvents (toluene, 99.9\% and isopropyl alcohol, 99.9\%) used in this work were ACS grade and purchased from Fisher Scientific.

For optical microscopy experiments, $n$-alkane samples were prepared on $500 \mu \mathrm{m}$ thick phosphorus doped silicon (110) wafers (University Wafer). For X-ray spectroscopy and microscope experiments, samples were prepared on $100 \mathrm{~nm}$ thick, $0.5 \times 0.5 \mathrm{~mm}$ low stress silicon nitride $\left(\mathrm{Si}_{3} \mathrm{~N}_{4}\right)$ windows (Norcada Inc.). 
Sample Preparation: Thin crystals of $n$-tetracontane $\left(n-\mathrm{C}_{40} \mathrm{H}_{82}\right), n$-dotriacontane $\left(n-\mathrm{C}_{32} \mathrm{H}_{66}\right)$ and $n$ octacosane $\left(n-\mathrm{C}_{28} \mathrm{H}_{58}\right)$ were prepared by solution casting.[25, 26] $n$-Alkane molecules were dissolved in a suitable solvent with a mass/volume ratio of $1.0 \mathrm{mg} / 2.00 \mathrm{ml}$ and stirred at $50{ }^{\circ} \mathrm{C}$. A drop of a solution was placed on the substrate and solvent evaporation occurred. The substrate temperature during evaporation, the solvent and substrate were optimized in order to isolate orthorhombic or monoclinic polymorphs for a given $n$-alkane chain length (see Table $\mathbf{S 1}$ in Supplementary Material).

Optical and X-ray microscopy shows that these crystals are diamond or rectangular in shape; this indicates that the $(\mathrm{a}, \mathrm{b})$ unit cell axes of the crystals are oriented in the substrate plane. Single crystals with straight facet edges and well-defined facet angles could be found for all $n$-alkane samples. As discussed below (see §3.1), crystals were assigned to monoclinic or orthorhombic crystal structures based on measurement of interior angles. As the convention for defining the a and $b$ axes differs between the orthorhombic and monoclinic polymorphs (see Scheme 1a and 1b), the principal axis of the crystal shapes (long axis, X; short axis, Y) are used to describe the LDNEXAFS experimental geometry.

\subsection{Optical Microscopy and X-ray Spectromicroscopy Characterization.}

The morphology of the $n$-alkane single crystals was characterized by optical microscopy (Nikon Eclipse ME600, with a Q-Imaging CCD camera) under bright field mode. Representative images are presented in the Supplementary Material, Figure S1.

NEXAFS imaging and spectroscopy were obtained at the Spectromicroscopy (SM) beamline (10ID-1, Apple II Elliptically Polarized Undulator) at the Canadian Light Source (CLS), using the ambient Scanning Transmission X-ray Microscope (a-STXM).[41] Spectra and images were acquired in a transmission mode. This presentation focuses on carbon $1 \mathrm{~s} \rightarrow \sigma^{*}{ }_{\mathrm{C}-\mathrm{H}}$ transitions (the "C-H" band; $287-288 \mathrm{eV}$ ), in order to highlight LD features that are expected to vary with crystal structure and chain length. STXM images used for quantitative measurement of the $n$-alkane internal angles were recorded in "point by point" mode, as this mode produced images with less distortion than "rapid scan" imaging mode.

Angle dependent NEXAFS spectra were measured by rotating the sample azimuthally, that is, around the photon propagation direction, while using a fixed electric field polarization vector. The SM beamline's elliptically polarized undulator (EPU) can produce arbitrarily inclined linear 
polarization. However, small variations in the degree of linear polarization have been observed in the proximity of the carbon dip for horizontal and vertically polarized radiation (see Figure S2 in the Supplementary Material). To control for this variable, a fixed horizontal polarization was used so that the degree of polarization would be constant for all measurements. Crystals were selected with their principal axes ( $\mathrm{X}$ or $\mathrm{Y}$ ) directed horizontally. Small misalignments of the crystal principal axes (see Figure S3 in the Supplementary Material) with respect to the horizontal linear polarization has a small effect on the relative intensity of features in the " $\mathrm{C}-\mathrm{H}$ " band.

The relatively high radiation sensitivity of $n$-alkane molecules[42] limits the permissible dose that can be used for NEXAFS measurements. Experimental conditions (energy point spacing, dwell time, etc.) were optimized to minimize radiation exposure. Image sequence[43] acquisition was used, so that the radiation was distributed over a larger sample volume, and so that the I and Io spectra could be acquired simultaneously. The STXM beam was defocused to $150 \mathrm{~nm}$ diameter during image sequence data acquisition. Fresh sample areas were used for each spectrum. The threshold for radiation damage was determined by examining the NEXAFS spectra in the carbon $1 \mathrm{~s}$ continuum $(300 \mathrm{eV})$ to test for mass loss and at the energy of the carbon $1 \mathrm{~s} \rightarrow \pi^{*} \mathrm{C}=\mathrm{C}$ transition $(285 \mathrm{eV})$ to test for the formation of $\mathrm{C}=\mathrm{C}$ double bonds.[42, 44] In the experimental conditions used, radiation damage was found to be minimal.

The calibration of the energy scale for the carbon 1s NEXAFS spectra was performed by introducing $\mathrm{CO}_{2}$ into the microscope chamber with the sample in place, allowing the NEXAFS spectrum of the alkane sample and $\mathrm{CO}_{2}$ gas to be recorded simultaneously. The monochromator energy scale was calibrated based on the two vibronic peaks of gaseous $\mathrm{CO}_{2}$ : carbon $1 \mathrm{~s} \rightarrow 3 \mathrm{~s}(v=0)$ and carbon $1 \mathrm{~s} \rightarrow 3 \mathrm{p}(\mathrm{v}=0)$ transitions, which were set to the value of $292.74 \mathrm{eV}$ and $294.96 \mathrm{eV}$ respectively, after Ma et al. $[8,45]$ As all spectra were recorded with in situ $\mathrm{CO}_{2}$ calibration, the spectra are distorted by the strong carbon $1 \mathrm{~s} \rightarrow \pi^{*}$ transition centred at $290.74 \mathrm{eV}$.

Transmission spectra were converted to optical density with Beer's law (OD $=-\ln \left(\mathrm{I} / \mathrm{I}_{0}\right)$. Data analysis were performed using aXis $2000,{ }^{40}$ and spectra are presented using Origin 7.5 software package. ${ }^{41}$ 


\section{Experimental Results}

\subsection{Crystal Characterization of $\boldsymbol{n}$-Alkanes.}

Optical microscopy (OM) was used to identify the shape (diamond or parallelogram) of the welldefined $n$-alkane crystals. Acute interior angles of each $n$-alkane crystal were calculated from high resolution point-by-point STXM images of $n$-alkane crystal. With the aid of literature,[29, 30, 34, $35,46-50]$ acute interior angles were used to determine the crystal structure of each $n$-alkane single crystal.

Scheme 1 presents the literature unit cell parameters, acute interior angles of the crystal polymorphs, schematics of the unit cells and the chain orientation within these cells, the molecular arrangement within the orthorhombic and monoclinic crystal, and the principal axes (long axis, $\mathrm{X}$ and short axis, Y) of the $n$-alkane single crystals used to obtain the NEXAFS spectra.[25, 26, 2931, 34-36, 46-53] Our measured acute internal angles agree with the values derived from literature orthorhombic and monoclinic crystal structures. Each $n$-alkane crystal examined in this work shows either an orthorhombic (acute interior angle $68 \pm 1^{\circ}$ ) or a monoclinic structure (acute interior angle $\left.74 \pm 1^{\circ}\right)$ when cast at room temperature $\left(25 \pm 1^{\circ} \mathrm{C}\right)$ or at lower temperature inside a refrigerator $\left(8 \pm 1{ }^{\circ} \mathrm{C}\right)$. Thinner orthorhombic crystals are favoured at lower casting temperature whereas thinner monoclinic crystals are favoured room temperature $\left(25 \pm 1^{\circ} \mathrm{C}\right)$.

Polarized optical microscopy images of $n$-tetracontane $\left(\mathrm{C}_{40} \mathrm{H}_{82}\right), n$-dotriacontane $\left(\mathrm{C}_{32} \mathrm{H}_{66}\right), n$ octacosane $\left(\mathrm{C}_{28} \mathrm{H}_{58}\right)$ crystals obtained from solution casting method are shown in Figure $\mathbf{S 1}$ in the Supplementary Materials.

\subsection{NEXAFS Measurements of $\boldsymbol{n}$-Alkanes.}

Figures 1 and 2 presents the variation in the "C-H" band of the carbon 1s NEXAFS spectra of $n$ alkanes with different chain lengths $\left(n-\mathrm{C}_{28} \mathrm{H}_{58}, n-\mathrm{C}_{32} \mathrm{H}_{66}\right.$ and $\left.n-\mathrm{C}_{40} \mathrm{H}_{82}\right)$ and with different crystal structures (orthorhombic and monoclinic) with the X-ray polarization directed along the principal crystal axes X (Figure 1) and Y (Figure 2), respectively. Spectra are normalized by matching the minimum and maximum intensity feature within the " $\mathrm{C}-\mathrm{H}$ " band.

The shape of the "C-H" band is similar when the X-ray linear polarization $(E)$ is directed along either the $\mathrm{X}$ or the $\mathrm{Y}$ principal axes, with only small differences between the different crystalline polymorphs. When the polarization $(E)$ is directed along the long $(\mathrm{X})$ axis, the first peak $\left(\sigma^{*}{ }_{\mathrm{C}-\mathrm{H}} /\right.$ 
$\left.R_{\|}\right)$is weaker and the second peak $\left(\sigma^{*} \mathrm{C}-\mathrm{H} / R_{\perp}\right)$ is stronger (See Figure 1). The relative intensity is inverted when the polarization $(E)$ is directed along the short $(\mathrm{Y})$ axis of each single crystal (see Figure 2). Detailed differences between these spectra will be discussed below.

\section{Discussion}

Each $n$-alkane $\left(n-\mathrm{C}_{40} \mathrm{H}_{82}, n-\mathrm{C}_{32} \mathrm{H}_{66}\right.$ and $\left.n-\mathrm{C}_{28} \mathrm{H}_{58}\right)$ examined in this study forms crystals with monoclinic and orthorhombic forms. The carbon 1s NEXAFS spectra of $n$-alkanes with these two crystal forms are similar to each other in shape. The greatest difference is between monoclinic and orthorhombic spectra, for the spectra recorded when the polarization was directed along the $\mathrm{Y}$ crystal axis (Figure 2).

The local structure (next neighbor, or chain to chain packing) in the monoclinic form is very similar to that in the orthorhombic form. The monoclinic crystal can be described by an orthorhombic subcell oriented along the $n$-alkane backbone; this sub-cell has nearly the same local arrangement and geometry as in the orthorhombic structure $\left(\beta=91.67^{\circ}\right.$ in the orthorhombic subshell in the monoclinic polymorph; $\beta=90^{\circ}$ in the orthorhombic polymorph).[30, 35] Differences in density, order and packing are known to affect the carbon 1s NEXAFS of polyethyene,[40] which is attributed to variations in Rydberg quenching and Rydberg - valence mixing with the degree of chain packing and density. As the local packing environment of both polymorphs is so similar, differences in Rydberg quenching / Rydberg - valence mixing are not expected. A similar spectroscopic interpretation can be used for both crystalline polymorphs.

It is intuitive to interpret the $\mathrm{LD}$ of the "C$-\mathrm{H}$ " band in terms of the sum of contributions from $n$ alkane molecules at the center and corner positions of the unit cell, as shown in Scheme 1c. The center and corner sites show a different inclination of their C-C-C backbone plane (setting angle) relative to the unit cell $(\mathrm{a}, \mathrm{b})$ vectors. Kawaguchi et al. measured a setting angle of $47^{\circ}$ between the C-C-C plane and the a axis for $n-\mathrm{C}_{32} \mathrm{H}_{66}$; [54] Zou et al estimated an angle of $\sim 48^{\circ}$ for $n$ $\mathrm{C}_{40} \mathrm{H}_{82}$ [25] from the crystallography data of Teare et al.[34] In the Pca2 and $\mathrm{P} 2{ }_{1} / \mathrm{a}$ space groups, the centre site is related to the corner site by a glide symmetry element, so the C-C-C setting angles are not necessarily orthogonal (although they would be orthogonal for a setting angle of $45^{\circ}$ ). 
Analysis of the LD in the NEXAFS spectra of orthorhombic crystals of $n$-tetracontane $\left(n-\mathrm{C}_{40} \mathrm{H}_{82}\right)$ and $n$-nonadecane $\left(n-\mathrm{C}_{19} \mathrm{H}_{40}\right)$ by Zou et al. showed that a simple molecular-geometry linear dichroism model does not work. [25] When Zou et al. examined dichroic ratios for the two "C-H band peaks, they found that these dichroic ratios differed from that expected from molecular geometry considerations. Zou et al. proposed that solid-state effects that reflect the symmetry of the crystal modify the LD, and postulated that a band structure interpretation is more appropriate to interpret the LD of these NEXAFS spectra.[25] An analysis of dichroic ratios was not performed in this work, as our use of in situ $\mathrm{CO}_{2}$ calibration prevents the spectroscopic continuum normalization needed for accurate difference calculations. As well, in the monoclinic species, the second $\mathrm{C}-\mathrm{H}$ band peak (the carbon $1 \mathrm{~s} \rightarrow \sigma^{*} \mathrm{C}-\mathrm{H} / R_{\perp}$ transition) will overlap with a backboneoriented transition discussed below; this transition is present in only one polarization. These facts, and unresolved vibronic[12] and nuclear motion[55] contributions obscure any simple analysis of these intensities and the calculation of linear dichroism differences.

The onset of the first peak (carbon $1 \mathrm{~s} \rightarrow \sigma^{*}{ }_{\mathrm{C}-\mathrm{H}} / R_{\|}$transition) appears at slightly lower energy in the spectra of the lower molecular weight monoclinic polymorphs $\left(n-\mathrm{C}_{28} \mathrm{H}_{58}\right.$ and $n-\mathrm{C}_{32} \mathrm{H}_{66}$; not for $n-\mathrm{C}_{40} \mathrm{H}_{82}$ ). The origin of this low energy onset is not immediately obvious. From modeling of defect structures, we expect the "C-H" band transitions to appear at lower energy for $n$-alkanes with thermally populated vibrational modes or with gauche defects.[55] However, and particularly for these shorter $n$-alkanes, the monoclinic polymorph is the thermodynamically stable form. Given the in situ calibration of the energy scale, we have a high confidence in the fidelity of this energy shift observation. Reflecting the band-structure postulate of Zou et al.[25] in modifying the LD of the carbon $1 \mathrm{~s} \rightarrow \sigma^{*} \mathrm{C}-\mathrm{H} / R_{\|}$and the carbon $1 \mathrm{~s} \rightarrow \sigma^{*}{ }_{\mathrm{C}-\mathrm{H}} / R_{\perp}$ transitions, a further modification of the $\sigma^{*} \mathrm{C}-\mathrm{H} / R_{\|}$core excited state unique to the monoclinic polymorph could be possible. However, as DFT simulations of NEXAFS spectra with periodic boundary conditions are nontrivial for non-orthorhombic unit cells, resolution of this question must be left to future work.

In the monoclinic polymorph, the $n$-alkane chain is inclined towards the short crystal axis ( $\mathrm{Y}$ in Scheme 1; $\beta=119.6^{\circ}$ instead of $\beta=90^{\circ}$ in the orthorhombic polymorph). As a result, NEXAFS spectra with the polarization component directed along the long axis ( $\mathrm{X}$ in Scheme 1b) will only have $\sigma^{*} \mathrm{C}-\mathrm{H} / R_{\|}$and $\sigma^{*}{ }_{\mathrm{C}-\mathrm{H}} / R_{\perp}$ character, while the component along the short axis (Y in Scheme 1b) will have some backbone TDM component. Fu and Urquhart demonstrated that a backbone- 
oriented transition is found $\sim 30 \mathrm{meV}$ above the second "C-H" band $\left(\sigma^{*}{ }_{\mathrm{C}-\mathrm{H}} / R_{\perp}\right.$; at $288.51 \mathrm{eV}$ in $n$-hexatriacontane, $n$ - $\mathrm{C}_{60} \mathrm{H}_{122}$.[21] Based on Fu's results, we expect a backbone-oriented transition to be observed in the monoclinic polymorph, but only when the polarization is directed along the short crystal axis, Y. Further, as the optical orbital for this transition is largely within the molecule itself, it should not be affected by the band structure / intermolecular effects proposed by Zou et $a l$. for the carbon $1 \mathrm{~s} \rightarrow \sigma^{*} \mathrm{C}-\mathrm{H} / R_{\|}$and carbon $1 \mathrm{~s} \rightarrow \sigma^{*}{ }_{\mathrm{C}-\mathrm{H}} / R_{\perp}$ transitions.[25] The intensity of this transition will be $\sim 24 \%$ of that when the polarization is completely directed along the $n$-alkane backbone (applying equation $1, \beta=119.6^{\circ}$ gives a backbone angle of $60.4^{\circ}$ from the polarization vector). This higher energy backbone-oriented component will be only visible in the "C-H" band for the Y-axis polarized spectra of the monoclinic polymorph. This is precisely the difference observed as a higher energy shoulder in Figure 2 (Y-axis polarized) for the monoclinic spectra $(\sim 288 \mathrm{eV})$ and absent in Figure 1 (X-axis polarized). We note that the comparison of the spectra of the orthorhombic and monoclinic $n-\mathrm{C}_{28} \mathrm{H}_{58}$ is not consistent with this trend; an exploration of the role of a thermally accessible defect mode uniquely present the orthorhombic species is the subject of temperature dependent NEXAFS studies now underway.[55]

\section{Conclusions}

This work presents the linear dichroism (LD) in the carbon 1s NEXAFS spectra of a series of $n$ alkanes ( $n-\mathrm{C}_{28} \mathrm{H}_{58} n-\mathrm{C}_{32} \mathrm{H}_{66}$ and $n-\mathrm{C}_{40} \mathrm{H}_{82}$ ), with orthorhombic and monoclinic structures. The key structural difference between the monoclinic and orthorhombic polymorphs is the inclination of the monoclinic unit cell ( $\beta=119.6^{\circ}$ versus $90^{\circ}$ in the orthorhombic structure), towards the short axis of the monoclinic single crystals. This inclination leads to a contribution from a backboneoriented transition in the "C-H" band, as observed by Fu et al.[21] This contribution appears as a high energy shoulder in the "C-H" band, but only for spectra with the polarization directed along the short axis $(Y)$ of the monoclinic crystal. These results show the importance of detailed crystal structure considerations when interpreting the NEXAFS spectra of single crystals. 


\section{ACKNOWLEDGMENTS}

SGU is supported by NSERC (Canada) and Canadian Foundation for Innovation. This research was carried in Soft Microscopy (SM) beamline at the Canadian Light Source (CLS) and it is supported by the Natural Sciences and Engineering Research Council of Canada, the National Research Council Canada, the Canadian Institutes of Health Research, the Province of Saskatchewan, Western Economic Diversification Canada, and the University of Saskatchewan. We thank Jianfeng Zhu for the helpful discussions. 


\section{Scheme Captions}

Scheme 1. Schematic diagram for the chain length orientation, published unit cell parameters and internal angles for (a) orthorhombic and (b) monoclinic structure of $\mathrm{C}_{40} \mathrm{H}_{82},[30,34,49]$ (c) the molecular arrangement in the orthorhombic and monoclinic polymorph, viewed within the $(a, b)$ plane (vertices are carbon; terminal positions are hydrogen) and (d) principal crystal axes for the $n$-alkane single crystals used in NEXAFS measurements.

Scheme 2. Schematic diagram of the TDM for the a.) first " $\mathrm{C}-\mathrm{H}$ " band transition (carbon $1 \mathrm{~s} \rightarrow$ $\sigma^{*} \mathrm{C}-\mathrm{H} / R_{\|}$) and the b.) second "C-H" band transition (carbon $1 \mathrm{~s} \rightarrow \sigma^{*} \mathrm{C}-\mathrm{H} / R_{\perp}$ transition), relative to a representative $n$-alkane chain ( $n$-pentane). Parallel and perpendicular are directions relative to the C-C-C plane; both perpendicular to the $n$-alkane backbone.

\section{Figure Captions}

Figure 1. Carbon 1s NEXAFS spectra of the " $\mathrm{C}-\mathrm{H}$ " band for $n$-alkanes $\left(n-\mathrm{C}_{40} \mathrm{H}_{82}, n-\mathrm{C}_{32} \mathrm{H}_{66}\right.$, and $n-\mathrm{C}_{28} \mathrm{H}_{58}$ ) with orthorhombic (black) monoclinic (red) crystal structure with X-ray linear horizontal polarization directed along long $(\mathrm{X})$ crystal axis.

Figure 2. Carbon 1s NEXAFS spectra of the " $\mathrm{C}-\mathrm{H}$ " band for $n$-alkanes $\left(n-\mathrm{C}_{40} \mathrm{H}_{82}, n-\mathrm{C}_{32} \mathrm{H}_{66}\right.$, and $n-\mathrm{C}_{28} \mathrm{H}_{58}$ ) with orthorhombic (black) monoclinic (red) crystal structure with X-ray linear horizontal polarization directed along short (Y) crystal axis. An asterisk indicates the backbone oriented transition in the NEXAFS spectrum of $n-\mathrm{C}_{40} \mathrm{H}_{82}$ and $n-\mathrm{C}_{32} \mathrm{H}_{66}$ ). 


\section{References}

[1] E.G. Rightor, S.G. Urquhart, A.P. Hitchcock, H. Ade, A.P. Smith, G.E. Mitchell, R.D. Priester, A. Aneja, G. Appel, G. Wilkes, W.E. Lidy, Identification and Quantitation of Urea Precipitates in Flexible Polyurethane Foam Formulations by X-ray Spectromicroscopy, Macromolecules 35 (2002) 5873-5882.

[2] J. Lipton-Duffin, J.A. Miwa, S.G. Urquhart, G. Contini, A. Cossaro, L. Casalis, J.V. Barth, L. Floreano, A. Morgante, F. Rosei, Binding Geometry of Hydrogen-Bonded Chain Motif in SelfAssembled Gratings and Layers on Ag(111), Langmuir 28 (2012) 14291-14300.

[3] S.E. Qaqish, S.G. Urquhart, U. Lanke, S.M.K. Brunet, M.F. Paige, Phase Separation of Palmitic Acid and Perfluorooctadecanoic Acid in Mixed Langmuir-Blodgett Monolayer Films, Langmuir 25 (2009) 7401-7409.

[4] G.R.S. Iyer, J. Wang, G. Wells, M.P. Bradley, F. Borondics, Nanoscale Imaging of Freestanding Nitrogen Doped Single Layer Graphene, Nanoscale 7 (2015) 2289-2294.

[5] A.P. Hitchcock, V. Berejnov, V. Lee, M. West, V. Colbow, M. Dutta, S. Wessel, Carbon Corrosion of Proton Exchange Membrane Fuel Cell Catalyst Layers Studied by Scanning Transmission X-ray Microscopy, Journal of Power Sources 266 (2014) 66-78.

[6] R. Meier, M. Schindler, P. Müller-Buschbaum, B. Watts, Residual Solvent Content in Conducting Polymer-Blend Films Mapped with Scanning Transmission X-ray Microscopy, Physical Review B 84 (2011) 174205.

[7] J. Zhong, H. Zhang, X. Sun, S.T. Lee, Synchrotron Soft X-ray Absorption Spectroscopy Study of Carbon and Silicon Nanostructures for Energy Applications, Advanced Materials 26 (2014) 7786-7806.

[8] O. Dhez, H. Ade, S.G. Urquhart, Calibrated NEXAFS Spectra of Some Common Polymers, Journal of Electron Spectroscopy and Related Phenomena 128 (2003) 85-96.

[9] H. Ade, S. Urquhart, NEXAFS Spectroscopy and Microscopy of Natural and Synthetic Polymers, Chemical Applications Of Synchrotron Radiation: Part I: Dynamics and VUV SpectroscopyPart II: X-Ray Applications, World Scientific2002, pp. 285-355.

[10] A. Gainar, J.S. Stevens, C. Jaye, D.A. Fischer, S.L. Schroeder, NEXAFS Sensitivity to Bond Lengths in Complex Molecular Materials: A Study of Crystalline Saccharides, The Journal of Physical Chemistry B 119 (2015) 14373-14381.

[11] J. Stohr, NEXAFS spectroscopy, Springer-Verlag Berlin1992.

[12] S.G. Urquhart, R. Gillies, Rydberg- Valence Mixing in the Carbon 1s Near-Edge X-ray Absorption Fine Structure Spectra of Gaseous Alkanes, The Journal of Physical Chemistry A 109 (2005) 2151-2159.

[13] S.G. Urquhart, R. Gillies, Matrix Effects in the Carbon 1 s Near Edge X-ray Absorption Fine Structure Spectra of Condensed Alkanes, The Journal of Chemical Physics 124 (2006) 234704.

[14] A. Ney, V. Ney, K. Ollefs, D. Schauries, F. Wilhelm, A. Rogalev, X-Ray Linear Dichroism: An Element-Selective Spectroscopic Probe for Local Structural Properties and Valence, Journal of Surfaces and Interfaces of Materials 2 (2014) 14-23.

[15] G. Rossi, F. d'Acapito, L. Amidani, F. Boscherini, M. Pedio, Local Environment of Metal Ions in Phthalocyanines: K-edge X-ray Absorption Spectra, Physical Chemistry Chemical Physics 18 (2016) 23686-23694. 
[16] H. Marchetto, T. Schmidt, U. Groh, F.C. Maier, P.L. Lévesque, R.H. Fink, H.-J. Freund, E. Umbach, Direct Observation of Epitaxial Organic Film Growth: Temperature-Dependent Growth Mechanisms and Metastability, Physical Chemistry Chemical Physics 17 (2015) 29150-29160. [17] J.E. Baio, C. Jaye, D.A. Fischer, T. Weidner, High-Throughput Analysis of Molecular Orientation on Surfaces by NEXAFS Imaging of Curved Sample Arrays, ACS Combinatorial Science 16 (2014) 449-453.

[18] S.N. Patel, G.M. Su, C. Luo, M. Wang, L.A. Perez, D.A. Fischer, D. Prendergast, G.C. Bazan, A.J. Heeger, M.L. Chabinyc, E.J. Kramer, NEXAFS Spectroscopy Reveals the Molecular Orientation in Blade-Coated Pyridal[2,1,3]thiadiazole-Containing Conjugated Polymer Thin Films, Macromolecules 48 (2015) 6606-6616.

[19] M. Masnadi, S.G. Urquhart, Indirect Molecular Epitaxy: Deposition of n-Alkane Thin Films on $\mathrm{Au}$ Coated $\mathrm{NaCl}$ (001) and HOPG (0001) Surfaces, Langmuir 29 (2013) 6302-6307.

[20] S.G. Urquhart, U.D. Lanke, J. Fu, Characterisation of Molecular Orientation in Organic Nanomaterials by X-ray Linear Dichroism Microscopy, International Journal of Nanotechnology 5 (2008) 1138-1170.

[21] J. Fu, S.G. Urquhart, Linear Dichroism in the X-Ray Absorption Spectra of Linear nAlkanes, The Journal of Physical Chemistry A 109 (2005) 11724-11732.

[22] J. Thieme, G. Schmahl, D. Rudolph, E. Umbach, X-Ray Microscopy and

Spectromicroscopy: Status Report from the Fifth International Conference, Würzburg, August 19-23, 1996, Springer Science \& Business Media2013.

[23] G.P. Hastie, J. Johnstone, K.J. Roberts, D. Fischer, Examination of the Structure and Melting Behaviour of Thin Film n-Alkanes using Ultra-Soft Polarised Near-Edge X-ray Absorption Spectroscopy, Journal of the Chemical Society, Faraday Transactions 92 (1996) 783789.

[24] J. Fu, S.G. Urquhart, Effect of Chain Length and Substrate Temperature on the Growth and Morphology of n-Alkane Thin Films, Langmuir 23 (2007) 2615-2622.

[25] Y. Zou, T. Araki, G. Appel, A.L.D. Kilcoyne, H. Ade, Solid State Effects in the NEXAFS Spectra of Alkane-Based van der Waals Crystals: Breakdown of Molecular Model, Chemical Physics Letters 430 (2006) 287-292.

[26] S. Swaraj, H. Ade, Differences in NEXAFS of odd/even long chain n-alkane crystals, Journal of Electron Spectroscopy and Related Phenomena 191 60-64.

[27] M. Masnadi, S.G. Urquhart, Effect of Substrate Temperature on the Epitaxial Growth of Oriented n-Alkane Thin Films on Graphite, Langmuir 28 (2012) 12493-12501.

[28] O. Endo, H. Ozaki, R. Sumii, K. Amemiya, M. Nakamura, N. Kosugi, Orientation of nalkane in thin films on graphite (0001) studied using C K-NEXAFS, Journal of Electron Spectroscopy and Related Phenomena 184 (2011) 257-260.

[29] M.G. Broadhurst, An Analysis of the Solid Phase Behavior of the Mormal Paraffins, Journal of Research of the National Bureau of Standards 66 (1962) 241-249.

[30] M. Plomp, W. Van Enckevort, P. Van Hoof, C. Van De Streek, Morphology of and Dislocation Movement in n-C 40 H 82 Paraffin Crystals Grown from Solution, Journal of Crystal Growth 249 (2003) 600-613.

[31] R. Boistelle, B. Simon, G. Pepe, Polytypic structures of n-C28H58 (octacosane) and nC36H74 (hexatriacontane), Acta Crystallographica Section B: Structural Crystallography and Crystal Chemistry 32 (1976) 1240-1243.

[32] H. Kubota, F. Kaneko, T. Kawaguchi, M. Kawasaki, Polytypic Transition of nHexatriacontane During Solution Crystallization, Crystal Growth \& Design 4 (2004) 369-375. 
[33] H. Kubota, F. Kaneko, T. Kawaguchi, M. Kawasaki, Polytypic Transformation During Crystal Growth Monitored By Newly Developed Micro-FTIR System for Three-Dimensional Structural Studies, Journal of Crystal Growth 237 (2002) 373-378.

[34] P.W. Teare, The crystal structure of orthorhombic hexatriacontane, C36H74, Acta Crystallographica 12 (1959) 294-300.

[35] H.M.M. Shearer, V. Vand, The crystal structure of the monoclinic form of nhexatriacontant, Acta Crystallographica 9 (1956) 379-384.

[36] W.R. Turner, Normal Alkanes, Industrial \& Engineering Chemistry Product Research and Development 10 (1971) 238-260.

[37] D. Outka, J. Stöhr, J. Rabe, J. Swalen, H. Rotermund, Orientation of Arachidate Chains in Langmuir-Blodgett Monolayers on Si (111), Physical Review Letters 59 (1987) 1321.

[38] G. Hähner, M. Kinzler, C. Wöll, M. Grunze, M. Scheller, L. Cederbaum, Near Edge X-rayAbsorption Fine-Structure Determination of Alkyl-Chain Orientation: Breakdown of the “'Building-Block' Scheme, Physical Review Letters 67 (1991) 851.

[39] K. Weiss, H. Öström, L. Triguero, H. Ogasawara, M.G. Garnier, L.G.M. Pettersson, A. Nilsson, XPS and XAS investigation of condensed and adsorbed n-octane on a $\mathrm{Cu}(110)$ surface, Journal of Electron Spectroscopy and Related Phenomena 128 (2003) 179-191.

[40] A. Scholl, R. Fink, E. Umbach, G.E. Mitchell, S.G. Urquhart, H. Ade, Towards a detailed understanding of the NEXAFS spectra of bulk polyethylene copolymers and related alkanes, Chemical Physics Letters 370 (2003) 834-841.

[41] K.V. Kaznatcheev, C. Karunakaran, U.D. Lanke, S.G. Urquhart, M. Obst, A.P. Hitchcock, Soft X-ray spectromicroscopy beamline at the CLS: Commissioning results, Nuclear Instruments and Methods in Physics Research Section A: Accelerators, Spectrometers, Detectors and Associated Equipment 582 (2007) 96-99.

[42] T. Coffey, S.G. Urquhart, H. Ade, Characterization of the effects of soft X-ray irradiation on polymers, Journal of Electron Spectroscopy and Related Phenomena 122 (2002) 65-78.

[43] J. Wirick, F. Zimba, Soft X-ray spectroscopy from image sequences with sub-100nm spatial resolution, Journal of Microscopy 197 (2000) 173-184.

[44] E.G. Rightor, A.P. Hitchcock, H. Ade, R.D. Leapman, S.G. Urquhart, A.P. Smith, G. Mitchell, D. Fischer, H.J. Shin, T. Warwick, Spectromicroscopy of poly (ethylene terephthalate): comparison of spectra and radiation damage rates in X-ray absorption and electron energy loss, The Journal of Physical Chemistry B 101 (1997) 1950-1960.

[45] Y. Ma, C.T. Chen, G. Meigs, K. Randall, F. Sette, High-resolution K-shell photoabsorption measurements of simple molecules, Physical Review A 44 (1991) 1848.

[46] X.Y. Liu, P. Bennema, On the morphology of normal alkane crystals with monoclinic structures: theory and observations, Journal of Applied Crystallography 26 (1993) 229-242. [47] S.R. Craig, G.P. Hastie, K.J. Roberts, J.N. Sherwood, Investigation into the structures of some normal alkanes within the homologous series $\mathrm{C} 13 \mathrm{H} 28$ to $\mathrm{C} 60 \mathrm{H} 122$ using high-resolution synchrotron X-ray powder diffraction, Journal of Material Chemistry 4 (1994) 977-981.

[48] D.L. Dorset, Crystal structure of an n-paraffin binary eutectic solid. An electron diffraction determination, The Journal of Physical Chemistry B 101 (1997) 4870-4874.

[49] S.C. Nyburg, J.A. Potworowski, Prediction of unit cells and atomic coordinates for the nalkanes, Acta Crystallographica Section B: Structural Crystallography and Crystal Chemistry 29 (1973) 347-352. 
[50] V. Chevallier, D. Petitjean, V. Ruffier-Meray, M. Dirand, Correlations between the crystalline long c-parameter and the number of carbon atoms of pure n-alkanes, Polymer 40 (1999) 5953-5956.

[51] B. Hubbard, Some Observations On The Optical Properties Of Long Chain Normal Paraffins, American Mineralogist 30 (1945) 645-672.

[52] J. van de Streek, P. Verwer, P. Bennema, E. Vlieg, On the influence of thermal motion on the crystal structures and polymorphism of even n-alkanes, Acta Crystallographica Section B: Structural Science 58 (2002) 677-683.

[53] A. Kawaguchi, S. Isoda, M. Ohara, K.-i. Katayama, Radiation-induced phase transition of paraffins, Bulletin of the Institute for Chemical Research 59 (1981) 284-292.

[54] A. Kawaguchi, M. Ohara, K. Kobayashi, Setting angle of molecular chains in polyethylene crystals, Journal of Macromolecular Science, Part B 16 (1979) 193-212.

[55] S.D. Perera, S. Shokatian, J. Wang, S.G. Urquhart, Temperature Dependence in the NEXAFS Spectra of n-Alkanes, The Journal of Physical Chemistry A (2018). 
(a) Orthorhombic

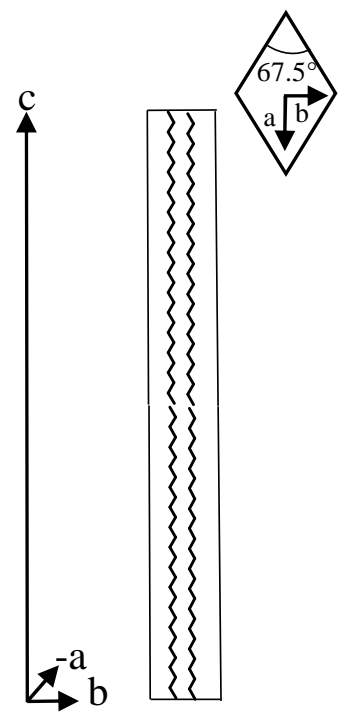

$\mathrm{Pca} 2_{1}, \mathrm{Z}=4$

$\mathrm{a}=7.42 \AA(\mathbf{X})$

$\mathrm{b}=4.96 \AA(\mathbf{Y})$

$\mathrm{c}=95.14 \AA$

$\alpha=\beta=\gamma=90^{\circ}$ (b) Monoclinic



$\mathrm{P} 2{ }_{1} / \mathrm{a}, \mathrm{Z}=2$

$\mathrm{a}=5.57 \AA(\mathbf{Y})$

$\mathrm{b}=7.42 \AA(\mathbf{X})$

$\mathrm{c}=48.35 \AA$

$\alpha=\gamma=90^{\circ}, \beta=119.6^{\circ}$ (c) Molecular arrangement

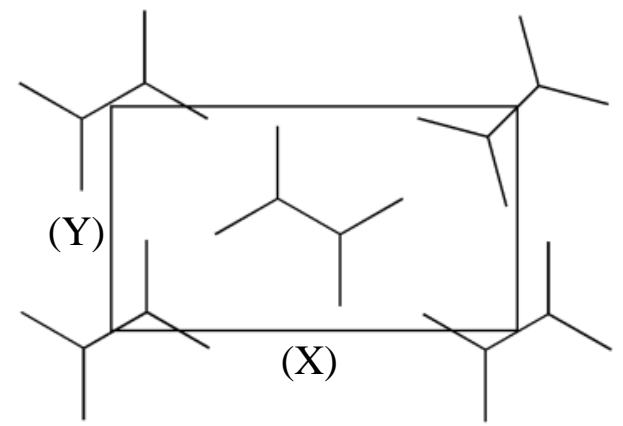

(d) Crystal Principle Axes

Long crystal axis (X)



Short crystal axis (Y)

\section{Scheme 1}




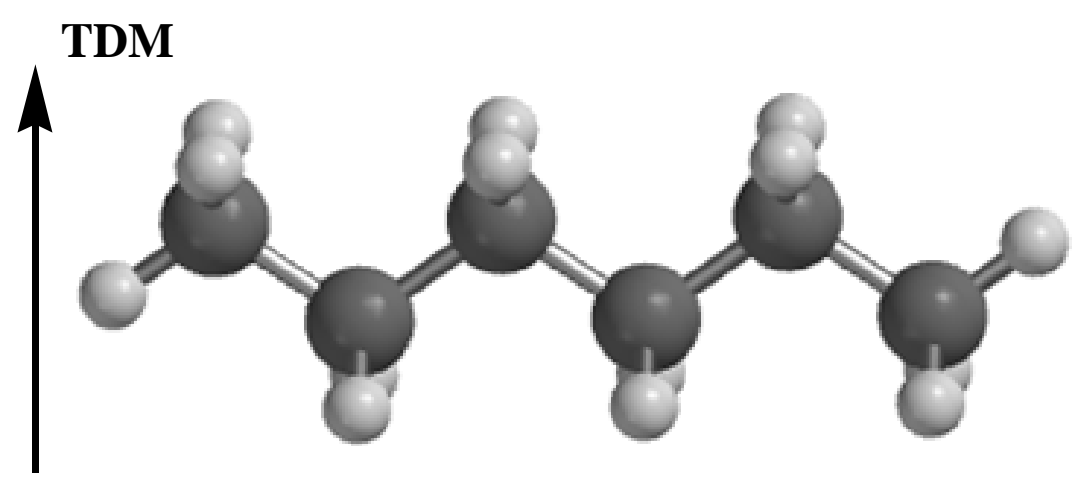

a.) $\sigma^{*}{ }_{\mathrm{C}-\mathrm{H}} / R_{\|}$

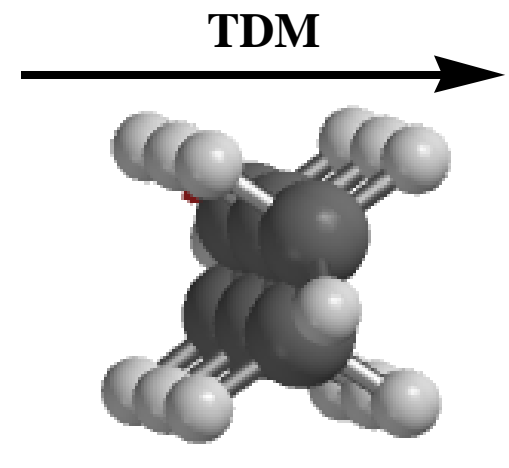

b.) $\sigma^{*}{ }_{\mathrm{C}-\mathrm{H}} / R_{\perp}$ 


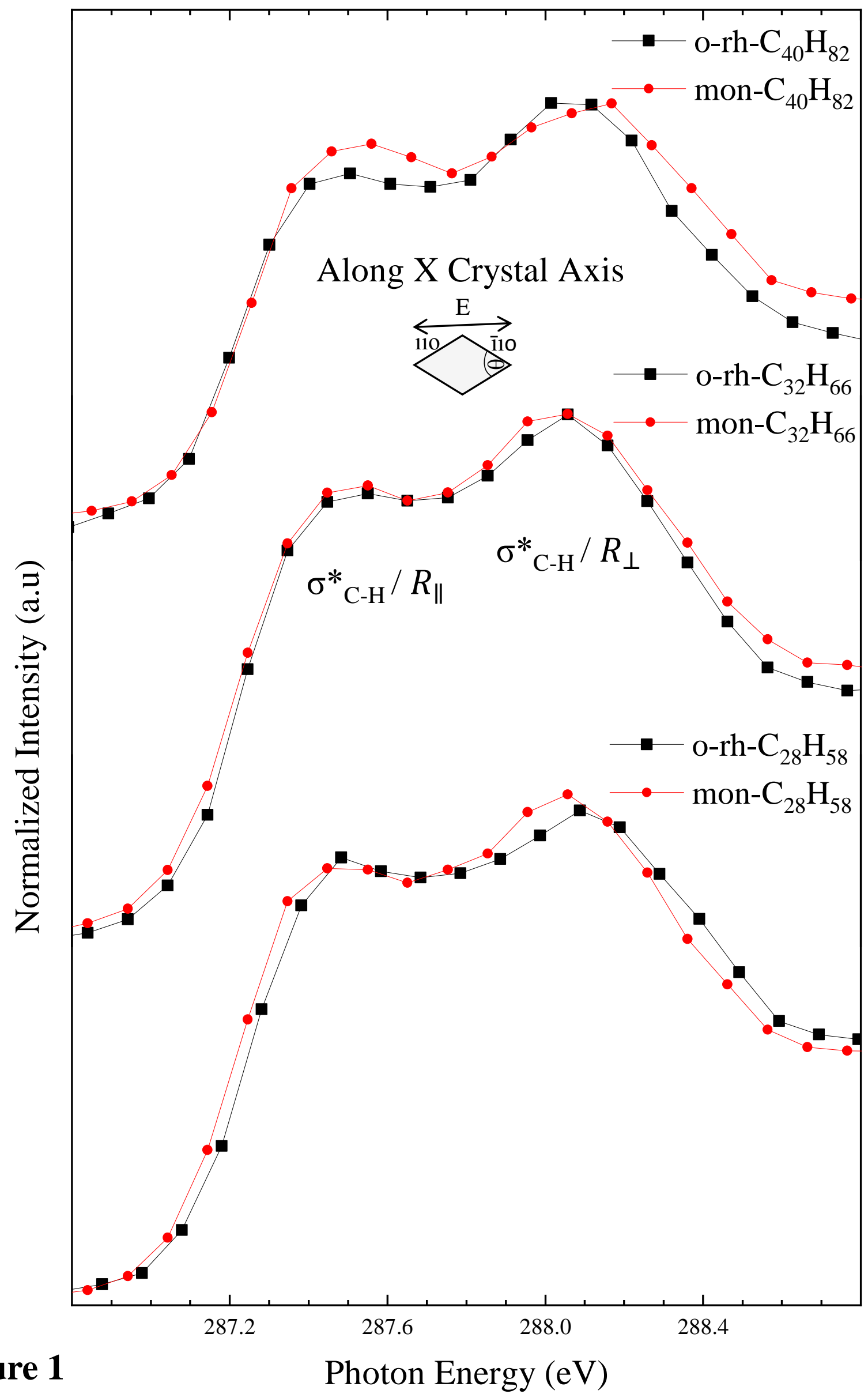

\section{Figure 1}

Photon Energy (eV) 




Figure 2

Photon Energy (eV) 


\section{Supplementary Material: Linear Dichroism in the NEXAFS \\ Spectra of Different Crystalline Polymorphs of $\mathbf{N}$-alkanes \\ Sahan D. Perera, Jian Wang, Stephen G. Urquhart}

Table S1 Optimum solution casting conditions for different $n$ alkane crystals

\begin{tabular}{|c|c|c|c|c|}
\hline n-Alkane & Type of Solvent & $\begin{array}{l}\text { Substrate for } \\
\text { Spectroscopic } \\
\text { Measurements }\end{array}$ & Crystal Structure & $\begin{array}{c}\text { *Solvent } \\
\text { Evaporation } \\
\text { Temperature }\end{array}$ \\
\hline \multirow[t]{2}{*}{$\mathrm{C}_{40} \mathrm{H}_{82}$} & \multirow[t]{2}{*}{ Toluene } & \multirow{2}{*}{$\begin{array}{c}100 \mathrm{~nm} \mathrm{Si}_{3} \mathrm{~N}_{4} \\
\text { window }\end{array}$} & Orthorhombic & $\sim 8^{\circ} \mathrm{C}$ \\
\hline & & & Monoclinic & $\sim 25^{\circ} \mathrm{C}$ \\
\hline \multirow[t]{2}{*}{$\mathrm{C}_{32} \mathrm{H}_{66}$} & \multirow[t]{2}{*}{ Isopropyl alcohol } & \multirow{2}{*}{$\begin{array}{c}100 \mathrm{~nm} \mathrm{Si}{ }_{3} \mathrm{~N}_{4} \\
\text { window }\end{array}$} & Orthorhombic & $\sim 8{ }^{\circ} \mathrm{C}$ \\
\hline & & & Monoclinic & $\sim 25^{\circ} \mathrm{C}$ \\
\hline \multirow[t]{2}{*}{$\mathrm{C}_{28} \mathrm{H}_{58}$} & \multirow[t]{2}{*}{ Isopropyl alcohol } & \multirow{2}{*}{$\begin{array}{c}100 \mathrm{~nm} \mathrm{Si}{ }_{3} \mathrm{~N}_{4} \\
\text { window }\end{array}$} & Orthorhombic & $\sim 8{ }^{\circ} \mathrm{C}$ \\
\hline & & & Monoclinic & $\sim 25^{\circ} \mathrm{C}$ \\
\hline
\end{tabular}

* Lower temperature $\sim 8{ }^{\circ} \mathrm{C}$ obtained inside the refrigerator and $\sim 25^{\circ} \mathrm{C}$ corresponds to the room temperature. 




Figure S1: Optical Microscopy (OM) images of (a) $n-\mathrm{C}_{28} \mathrm{H}_{58}$ (C28), (b) $n$ $\mathrm{C}_{32} \mathrm{H}_{68}$ (C32) and (c) $n-\mathrm{C}_{40} \mathrm{H}_{82}$ (C40) under bright field illumination. $\mathrm{M}$ and $\mathrm{O}$ are monoclinic (mon) and orthorhombic (o-rh) crystal structure. 




Figure S2: Carbon 1s NEXAFS spectra of $n$-tetracontane $\left(\mathrm{C}_{40} \mathrm{H}_{82}\right)$ recorded with left circularly polarized radiation for different orientations A and B of the same single crystal. 



Figure S3: Effect of misalignment of the $n$-alkane crystal (a) on the long (X) crystal axis and (b) on the short (Y) crystal axis to the carbon 1s NEXAFS spectra under linear horizontal polarization. 
(a) Along $X$ crystal ax is
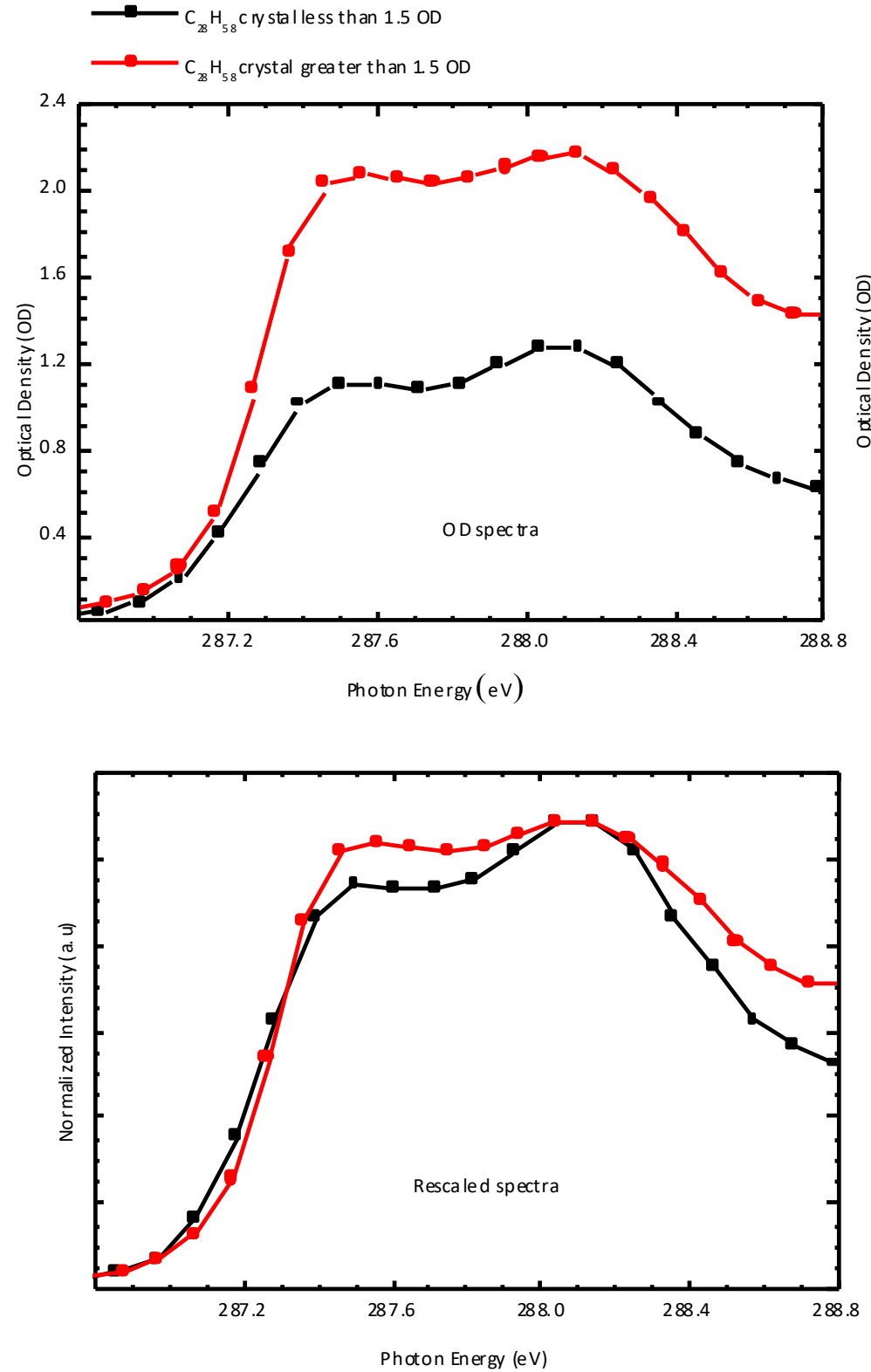

(b) Al ong Ycrystal a xis


Figure S4 Effect of crystal thickness on the carbon 1s NEXAFS spectra (a) along the long $(X)$ crystal axis and $(b)$ along the short $(Y)$ crystal axis under linear horizontal polarization. 